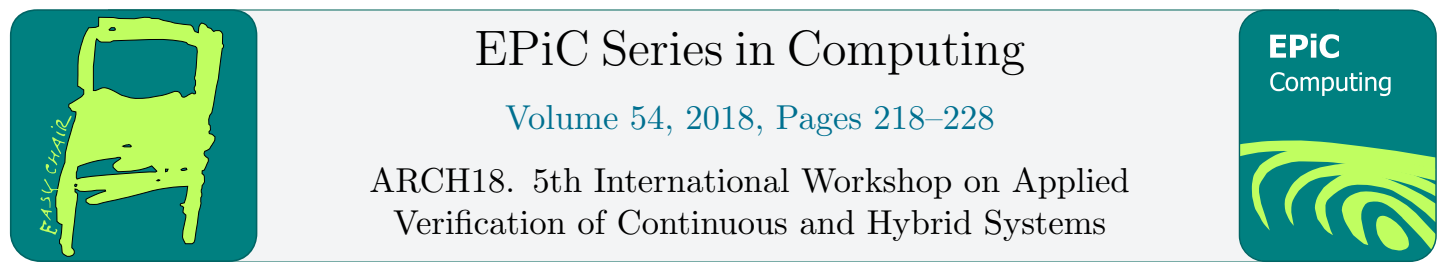

\title{
Space Debris Collision Detection using Reachability
}

\author{
Kerianne Hobbs ${ }^{1,2}$, Peter Heidlauf ${ }^{1}$, Alexander Collins ${ }^{1}$, and Stanley Bak ${ }^{3}$ \\ 1 Air Force Research Laboratory, Aerospace Systems Directorate \\ \{kerianne.hobbs, peter.heidlauf.1, alexander.collins.3\}@us.af.mil \\ 2 Georgia Institute of Technology \\ kerianne@gatech.edu \\ 3 Safe Sky Analytics \\ stanleybak@gmail.com
}

\begin{abstract}
Benchmark Proposal: Space debris tracking and collision prediction is a growing worldwide problem as more and more objects are placed into orbit. While traditional methods simulate particles with Gaussian uncertainty to make collision predictions, we instead analyze the problem from a reachability perspective. The problem appears to require methods capable of quickly analyzing high-dimensional nonlinear systems, but we take advantage multiple kinds of problem structure to show that reachability analysis may be viable for this problem. In particular we present an initial analysis approach that uses numerical simulation for reachability analysis, and interval arithmetic with AABB trees for fast collision detection. The analysis uses a variable size time step with a counter-example guided abstraction refinement (CEGAR) method to increase analysis speed without sacrificing accuracy. Our approach can analyze upwards of thousands of orbiting objects faster than real-time, where each object is subject to some initial state uncertainty.
\end{abstract}

\section{Introduction}

Historically, space situational awareness has been a combined effort by NASA, the Department of Defense (DoD) and the intelligence community. While the techniques used to predict satellite and debris conjunctions have been effective, they feature a very high false alarm rate. In addition, they are overwhelmed by the large number of objects, more than 100 objects that are regularly changing orbits, and high uncertainty in the locations of resident space objects. While 23,000 objects larger than $10 \mathrm{~cm}$ in diameter are regularly tracked by the U.S. government, 500,000 objects between 1 and $10 \mathrm{~cm}$ as well as more than 100 million objects smaller than 1 $\mathrm{cm}$ are predicted to exist [13]. The sheer number of objects presents many challenges in the n-to-n prediction of a conjunction between any two objects. In addition to the growing number of objects, the conjunction prediction problem is further complicated by slow ascent or descent

DISTRIBUTION A: Approved for public release; distribution unlimited. Case Number: 88ABW-2018-2108, completed on 23 Apr 2018. 
satellites and commercial satellite maintenance or inspection services that regularly change orbits. Furthermore, space surveillance can provide an accurate update on the positions and velocities of resident space objects once every few days, with a significant amount of uncertainty between observations. A variety of academic models have been used to predict spacecraft position over time, typically using simulation-based analysis [10, 1, 18, 2, 5].

Several orbit and rendezvous benchmarks have recently been proposed for linearized dynamics based on the Clohessy-Wiltshire Hill $(\mathrm{CWH})$ equations $[14,16,6]$. These dynamics are applicable in a small section of the orbit where linearization is reasonable. Nonlinear orbital dynamics has also been considered in the case of coaxial and coplanar orbits [17]. This work used hybridization $[3,12]$ to analyze the nonlinear dynamics and prove collision avoidance for the two-satellite case. Here, in contrast, we consider the more general orbit case, with different axes and different planes of orbit. Further, we aim to analyze thousands of orbiting objects concurrently.

What enables analysis for such high-dimensional systems is that the dynamics of orbiting objects are disjoint from one another, and so rather than analyzing a single high-dimensional system, we analyze thousands of low-dimensional systems and combine the results together. In the simplest case, each orbiting object can be modeled as a one-dimensional dynamical system, where the variable is the angle within the orbit, the true anomaly $\nu$. Verification is nonetheless challenging as the safety property requires a nonlinear mapping between each object's $\nu$ and a $3 \mathrm{~d}$ position in space, and then an $n$-to- $n$ collision check to be performed at every point in time.

We first present a nonlinear orbital dynamics model for the general case of noncoaxial and noncoplanar orbits in Section 2. Next, Section 3 describes the verification problem and proposes a preliminary verification approach based on simulation-based reachability analysis, fast collision-detection data structures, and CEGAR-based variable step size. The setup demonstrates that verification is feasible, although detailed comparisons and further optimizations are left for future work.

\section{Orbital Dynamics Modeling}

This section describes the basic motion of an object in orbit about Earth, at increasing levels of complexity. One way to define an orbit is by the $\mathrm{x}, \mathrm{y}$, and $\mathrm{z}$ components of the instantaneous position $\vec{r}$ and velocity $\vec{v}$ vectors. Alternatively, the orbit may be defined with six standard orbital elements: semi-major axis $a$, eccentricity $e$, true anomaly $\nu$, inclination $i$, right ascension of the ascending node $\Omega$, and argument of perigee $\omega$. We use the six parameters approach, which is described in this section. A summary of the variables to be used is in Table 1.

Several simplifying assumptions can be made to slowly build up to the complexity of the full collision prediction problem, and they are presented incrementally in this paper. First, the parameters of the satellite are assumed to be perfectly known (in reality there is a significant amount of uncertainty about the position and velocity). Second, the satellite is assumed to follow a perfect Kepler orbit (in reality there are several perturbing forces such as air drag in lower orbits, variations in gravity over the earth, and solar radiation pressure that may be accounted for with more complex modeling frameworks). The calculations are further simplified by propagating only one of the orbital elements $\nu$ in a perifocal reference frame $\mathcal{F}_{p}$ specific to each individual satellite's orbit. To compare the positions of satellites in different orbits, each individual orbit undergoes a linear transformation to an Earth-centered inertial (ECI) reference frame $\mathcal{F}_{i}$. The position of each satellite can then be calculated and compared in the common inertial reference frame. This process is described in more detail in this section. 
Table 1: Summary of Orbital Elements and Parameters

\begin{tabular}{|c|c|c|c|c|}
\hline Symbol & Units & Range & Name & Shown in Figure(s) \\
\hline$a$ & $\mathrm{~km}$ & {$\left[6371,1.5 \times 10^{6}\right]$} & semi-major axis & $1,3,4$ \\
\hline$e$ & - & {$[0,1]$} & eccentricity & 2 \\
\hline $\mathcal{F}_{i}$ & - & - & Earth-centered inertial reference frame & 5 \\
\hline $\mathcal{F}_{p}$ & - & - & perifocal reference frame & 1,3, and 4,5 \\
\hline$i$ & rad & {$[0, \pi]$} & inclination & 6 \\
\hline$\mu$ & $\mathrm{km}^{3} / \mathrm{s}^{2}$ & $3.986012 \times 10^{5}$ & geocentric gravitational parameter & - \\
\hline$\nu$ & $\mathrm{rad}$ & {$[0,2 \pi]$} & true anomaly & $1,3,4$, and 6 \\
\hline$\Omega$ & $\mathrm{rad}$ & {$[0,2 \pi]$} & right ascension of the ascending node & 6 \\
\hline$\omega$ & $\mathrm{rad}$ & {$[0,2 \pi]$} & argument of perigee & 4 and 6 \\
\hline$p$ & $\mathrm{~km}$ & {$\left[6371,1.5 \times 10^{6}\right]$} & semi-latus rectum & 1,3, and 4 \\
\hline$\vec{r}$ & $\mathrm{~km}$ & {$\left[6371,1.5 \times 10^{6}\right]$} & position vector & $1,3,4$, and 6 \\
\hline$\vec{v}$ & $\mathrm{~km} / \mathrm{s}$ & {$[7.8,11.6]$} & velocity vector & $1,3,4$, and 6 \\
\hline$\Omega$ & - & - & ascending node & 6 \\
\hline$\Upsilon$ & - & - & vernal equinox & 5 and 6 \\
\hline
\end{tabular}

\subsection{Orbits in a Plane}

Spacecraft motion may generally be described by conic sections (circles, ellipses, parabolas, and hyperbolas). Orbits are modeled as ellipses in a plane with the Earth at one focus, as shown in Figure 1. The orbit may be described in a perifocal reference frame $\mathcal{F}_{p}$, which is specific to the orientation of the orbit and centered on the center of the Earth, where $\hat{p}$ is a unit vector pointing from the center of the Earth to perigee (the shortest distance from the center of Earth to the edge of the ellipse defining the orbit), $\hat{q}$ is orthogonal within the plane and aligned with the semi-latus rectum, and $\hat{w}$ is perpendicular to the orbital plane completing the orthogonal set. The perifocal orbit name comes from its use of perigee ("peri") in defining the first axis and the use of orbital ellipse's focal point ("focal") where Earth resides as the origin. The ellipse's shape is defined by the semi-major axis $a$, which is the furthest distance from the center of the ellipse to the edge, and eccentricity $e$, which is a measure of how much the orbit deviates from a circular orbit. As shown in Figure 2, an eccentricity of zero corresponds to a circular orbit and an eccentricity of one corresponds a parabolic trajectory that escapes Earth orbit. The location of the satellite within the elliptical orbit is described by the true anomaly $\nu$, which is the angle from the unit vector pointing to perigee $\hat{p}$ and the position vector $\vec{r}$ pointing from the center of the Earth to the satellite, as shown in Figure 1. The velocity vector $\vec{v}$ of the orbit is tangential to the ellipse at the location of the satellite.

Also specified in the orbital frame is the semi-latus rectum, calculated from the semi-major axis $a$ and eccentricity $e$, as described by

$$
p=a\left(1-e^{2}\right) .
$$

The magnitude of the position vector $\vec{r}$, denoted $r$, which represents the distance from the center of the Earth to the satellite, changes throughout the orbit and is calculated using

$$
r=|\vec{r}|=\frac{p}{(1+e \cos (\nu))}
$$



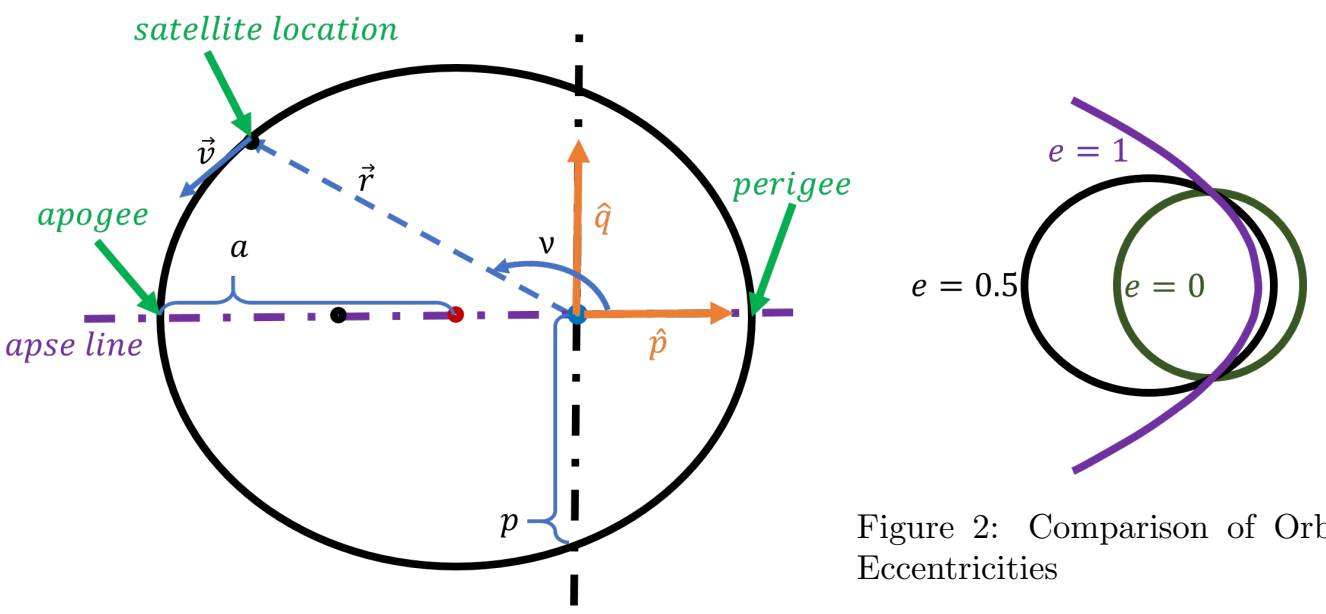

Figure 2: Comparison of Orbit Eccentricities

Figure 1: Perifocal Orbital Frame

Within the perifocal frame $\mathcal{F}_{p}$, the evolution of the spacecraft is modeled using Kepler's law of equal areas

$$
\dot{\nu}=\sqrt{\frac{\mu}{p^{3}}}(1+e \cos \nu)^{2} .
$$

In our proposed benchmark, each satellite has a single state variable corresponding to $\nu$, that evolves according to Equation 3. Equations 2 and 3 are essentially equations of one variable, the true anomaly $\nu$, because the semi-latus rectum $p$, eccentricity $e$, and geocentric gravitational parameter $\mu$ are assumed constant for the orbit. The position $\vec{r}_{p}$ of the spacecraft in the perifocal frame $\mathcal{F}_{p}$ is given by

$$
\vec{r}_{p}=\left[\begin{array}{lll}
r \cos \nu, & r \sin \nu, \quad 0
\end{array}\right]^{T} .
$$

The semi-major axis $a$, eccentricity $e$, and true anomaly $\nu$ are the first three of six orbital elements. Rather than starting with the full three-dimensional case including all six orbital elements, conjunction prediction algorithms can be evaluated with increasingly more complex combinations of orbits. The case of coaxial and coplanar orbits, as depicted in Figure 3, is the simplest model of two spacecraft orbits and may be defined by only three orbital elements: the semi-major axis $a$, the eccentricity $e$, and the true anomaly $\nu$. The apse line of each orbit is aligned and $\nu_{1}, \nu_{2}$ are measured from the same line, so the orbits share the same perifocal frame $\mathcal{F}_{p}$ and collisions may be predicted without transforming to a common reference frame.

Even if two satellites are in the same plane, if they do not share the same apse line, the perifocal frames of the orbits are no longer aligned as depicted in Figure 4. Therefore, any orbits that are not coaxial and coplanar must be transformed to a common reference frame to compare position vectors and predict collisions. The Earth-Centered Inertial frame provides a convenient common frame for comparisons between orbits and is described in the next subsection.

In additional to the semi-major axis $a$, eccentricity $e$, and true anomaly $\nu$, the case of noncoaxial but coplanar orbits requires the argument of perigee $\omega$ to predict collisions, as depicted in Figure 4. The argument of perigee $\omega$ is the angle within the plane that is measured from the ascending node $\Omega$ (the vector pointing from the center of the Earth to the point in 


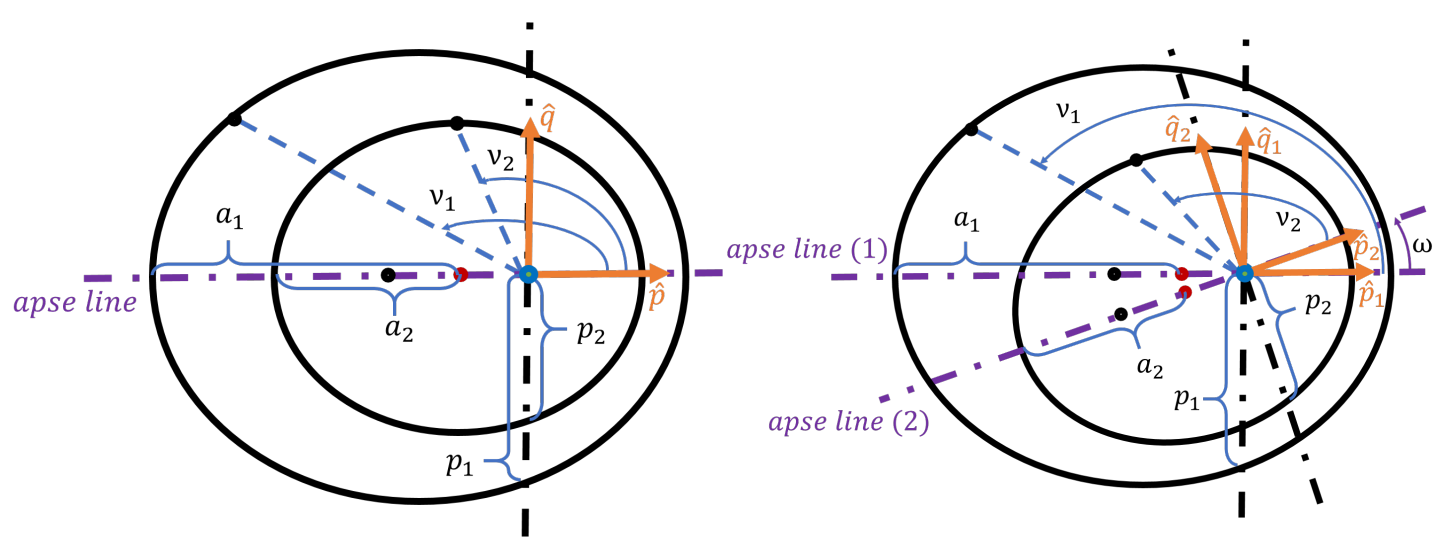

Figure 3: Coaxial and Coplanar Orbits

Figure 4: Noncoaxial, Coplanar Orbits

the three-dimensional orbit that crosses the Earth's equatorial plan from the southern to the northern hemisphere) to the vector pointing from the center of the Earth to perigee $\hat{p}$. The simplest model of two noncoaxial coplanar spacecraft orbits assumes that $\Omega$ is the same for both orbits, and only angle between perigee vectors differs $\left(\hat{p}_{1} \neq \hat{p}_{2}\right)$ differs by $\omega$.

\subsection{Orbits in an Inertial Frame}

An inertial frame is a reference frame that does not rotate or accelerate and is independent of the satellite's orbit. The Earth-Centered Inertial (ECI) frame $\mathcal{F}_{i}$ provides a convenient common frame for comparisons between orbits and its usefulness becomes more evident when noncoplanar orbits are compared for conjunction prediction.

The difference between the ECI frame $\mathcal{F}_{i}$ and the perifocal frame $\mathcal{F}_{p}$, shown in Figure 5 is defined by the argument of perigee $\omega$ as previously defined, as well as the orbital inclination $i$ and right ascension of the ascending node $\Omega$. The inclination $i$ is the angle from the equatorial plane to the orbital plane at the ascending node $\Omega$. The right ascension of the ascending node $\Omega$ is the angle from the vernal equinox $\Upsilon$ (vector from Earth's center to the Sun when aligned with the equatorial plane on the vernal equinox in the Northern Hemisphere) counter clockwise to the ascending node $\Omega$. These orbital elements are visualized in Figure 6 . The ECI frame's $\hat{i}$ axis is aligned with the vernal equinox $\Upsilon$, the $\hat{j}$ direction is orthogonal to the $\hat{i}$ axis so that together they span Earth's equatorial plane, and the $\hat{k}$ axis is aligned with Earth's spin axis.

Translating the motion from the inertial frame $\mathcal{F}_{i}$ to the orbital frame $\mathcal{F}_{p}$ is accomplished with a rotation matrix $R_{p i}$, derived from a sequence of Euler angle rotations. As shown in 7, Euler angles rotations rotate an orthogonal reference frame about one axis, resulting in the rotation matrices in Table 2.

The typical spacecraft convention is classic 3-1-3 Euler angle rotation sequence [19]. The first rotation is about the z-axis by the right ascension of the ascending node $\Omega$, followed by a rotation about the intermediate x-axis by the orbital inclination $i$, and finally a rotation about the second intermediate z-axis by the argument of perigee $\omega$. There is a singularity when 


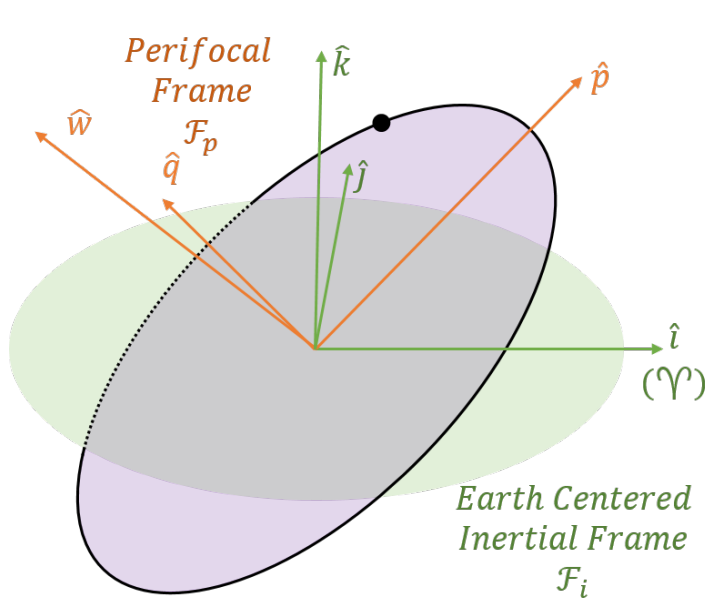

Figure 5: Orbital and Inertial Reference Frames

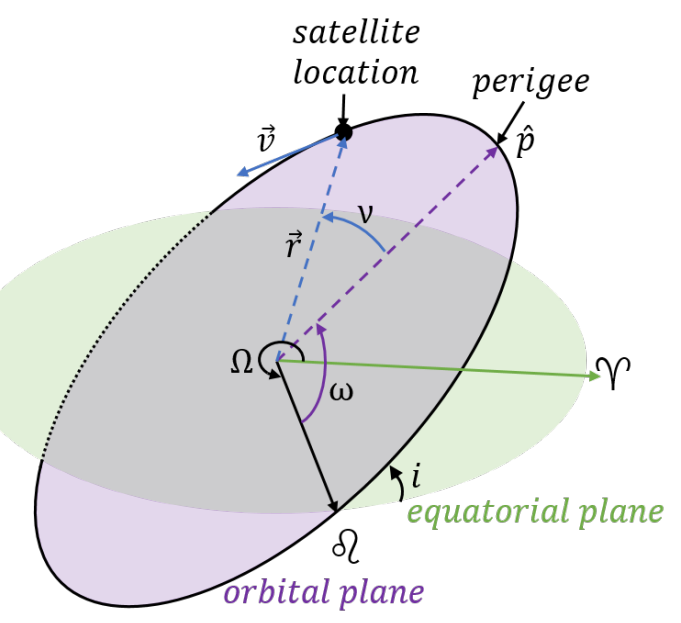

Figure 6: Orbits in the Inertial Frame

the inclination $i$ is 0 because the first and third rotations are about the same axis. Due to the properties of rotation matrices, going from the perifocal frame to the inertial frame is the transpose of the inertial to perifocal frame rotation $\left(R_{i p}=R_{p i}^{T}\right)$. The calculation is done with the linear transformation

$$
\vec{r}_{i}=R_{i p} \vec{r}_{p}=\left[R_{3}(\omega) R_{1}(i) R_{3}(\Omega)\right]^{T} \vec{r}_{p}=R_{3}^{T}(\Omega) R_{1}^{T}(i) R_{3}^{T}(\omega) \vec{r}_{p},
$$

where

$$
R_{i p}=\left[\begin{array}{ccc}
c \Omega c \omega-s \Omega c i s \omega & -c \Omega s \omega-s \Omega c i c \omega & s i s \Omega \\
s \Omega c \omega+c \Omega c i s \omega & -s \Omega s \omega+c \Omega c i c \omega & -c \Omega s i \\
s i s \omega & s i c \omega & c i
\end{array}\right]
$$

and $c$ is an abbreviation for cosine and $s$ is an abbreviation for sine. In inertial frame $\mathcal{F}_{i}$, the $x, y$, and $z$ components resulting from the rotation matrix $R_{i p}$ are

$$
\begin{aligned}
x_{\mathrm{i}} & =[c \Omega c \omega-s \Omega c i s \omega] x_{p}+[-c \Omega s \omega-s \Omega c i c \omega] y_{p}+[s i s \Omega] z_{p}, \\
y_{\mathrm{i}} & =[s \Omega c \omega+c \Omega c i s \omega] x_{p}+[-s \Omega s \omega+c \Omega c i c \omega] y_{p}+[-c \Omega s i] z_{p}, \text { and } \\
z_{\mathrm{i}} & =[\operatorname{sis} \omega] x_{p}+[\operatorname{sic} \omega] y_{p}+[c i] z_{p} .
\end{aligned}
$$

\section{Satellite Conjunction Verification}

In this section, we first state the satellite conjunction verification problem. Then, we describe our solution approach which takes advantage of several types of problem structure. Finally, we evaluate the scalability of the proposed method. 


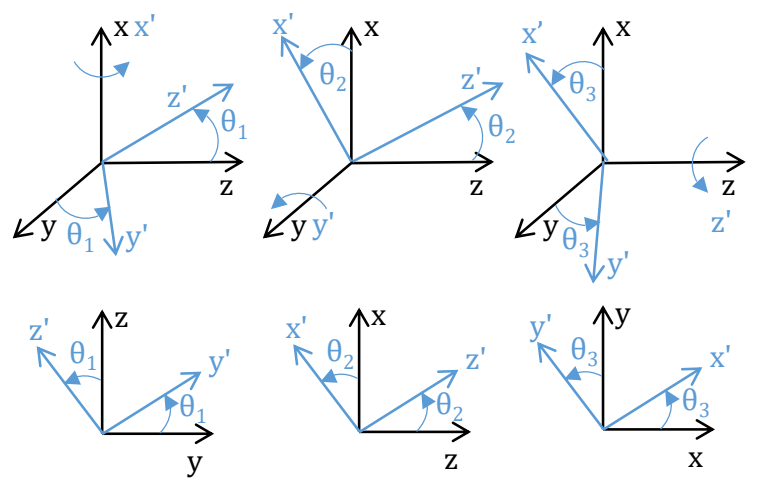

Figure 7: Euler Angle Rotations
Table 2: Euler Angle Rotation Descriptions and Matrices

\begin{tabular}{llll}
\hline Symbol Description & \multicolumn{1}{l}{ Rotation Matrix } \\
\hline \hline$R_{1}(\theta)$ & x-axis rotation & {$\left[\begin{array}{ccc}1 & 0 & 0 \\
0 & \cos \theta & \sin \theta \\
0 & -\sin \theta & \cos \theta\end{array}\right]$} \\
$R_{2}(\theta)$ & y-axis rotation & {$\left[\begin{array}{ccc}\cos \theta & 0 & -\sin \theta \\
0 & 1 & 0 \\
\sin \theta & 0 & \cos \theta\end{array}\right]$} \\
$R_{3}(\theta)$ & z-axis rotation & {$\left[\begin{array}{ccc}\cos \theta & \sin \theta & 0 \\
-\sin \theta & \cos \theta & 0 \\
0 & 0 & 1\end{array}\right]$}
\end{tabular}

\subsection{Problem Description}

The space debris collision detection problem is to prove that no two satellites collide (get within $\Delta$ distance) at any point in time given a list of initial parameters for $n$ satellites and a time bound. We consider the simplest case where satellite parameters are known constants, and the only uncertainty arises from the satellite angle on its orbit, the true anomaly $\nu$, which has dynamics defined by Equation 3. We want to analyze $n$-to- $n$ collision avoidance on thousands satellites, and the proof of safety should be done in dense time. Collisions are considered to occur when two satellites get within some collision radius of each other. For simplicity, we use the infinity norm for this (boxes are used for collision detection).

\subsection{Solution Approach}

Initially, this problem appears to be an $n$-dimensional nonlinear system, where $n$ is the number of orbiting objects, which can be on the order of 1000. Such a system is unlikely to be solved by off-the-shelf reachability methods that can only scale to a dozen or so state variables [8]. In order to analyze this system, we must leverage the problem structure.

Problem Structure. The first type of structure we use is that the dynamics is disjoint. Satellites move independently of each other when they orbit, and so the state variables do not interact. The derivative of each satellite's $\nu$ variable only depends on itself, and no other state variable. This allows us to convert the $n$-dimensional reachability problem into $n$ onedimensional reachability problems, and then recombine the reachable sets together after computation based on common notions of time [11].

The second type of structure used is that the decomposed problems are all one-dimensional. Due to the continuity of trajectories of the system, if no states from the boundaries of the initial set reach an unsafe state, then no states from the whole initial set (including the interior) can reach an unsafe state [9]. To do this, a trajectory would have to pass through a state that was reached by a boundary point, because trajectories must be continuous. For a high-dimensional system, this does not help too much since the boundary of an $n$-dimensional set will be an $n-1$ 
dimensional set, that still contains an infinite number of points. In the case of a 1-d system, however, the boundary of an initial set of states, an interval, is just the lower and upper bounds of the interval. This means that we only need to analyze trajectories from the lower and upper bounds and assure they are safe.

Numerical Simulation. Since we only analyze specific trajectories of a system, we take a numerical verification approach and assume that numerical simulations can produce accurate system behaviors. Using an off-the-shelf Runge-Kutta numerical simulator (scipy.integrate.RK45), we simulate the lower and upper bounds of $\nu$ to predict the timeevolved system states. This also can be used for analyzing intervals of time. Since orbits do not change direction, we can get the range of reachable $\nu$ values in an interval of time by sampling the endpoints of the range at the start of the time interval and at the end of the time interval, and taking the minimum and maximum over all four values.

Nonlinear Safety Property. A further challenge with this model is that the safety property is not expressed in terms of $\nu$, but requires converting $\nu$ to Cartesian $x, y$ and $z$ coordinates before doing a distance check. This is done according to Equation 7, which involves computing sine and cosine of $\nu$ as well as some of satellite orbit parameters.

To soundly convert from ranges of $\nu$ values to ranges of $x, y$ and $z$ coordinates, we use interval analysis methods [15]. Other methods, such as Taylor models (or affine arithmetic) could be considered. However, checking for collisions between two Taylor models is more difficult than checking for collisions between two boxes (the result of interval arithmetic), and computing with Taylor models may take more time. In our implementation, the bottleneck was actually the interval arithmetic computation, so we did not experiment with the more complicated methods (although they may allow one to take larger time steps, this could be a direction for future research).

Since we do the conversion using interval arithmetic, the set we use to check for collisions will always be a box, with the property that as the $\nu$ uncertainty tends towards zero, the volume of the output box will also tend towards zero.

The collision check needs to be done in an $n$-to- $n$ fashion. We considered three ways to implement this check: exhaustive search, k-d trees, and AABB trees.

The exhaustive search method of conjunction prediction evaluates the distance between every pair of objects. If using an off-the-shelf reachability tool, this is likely the method that would be used. We can take advantage of symmetry in the distance check in order to reduce the number of required checks, but we still expect this method to scale quadratically. The number of required checks with this approach is $\frac{n(n+1)}{2}$.

However, since we know our problem deals with 3-d spatial coordinates, we can leverage better data structures to reduce the number of checks in the safety condition. The first idea was to use $\mathrm{k}$-d trees. A k-d tree is a binary structure in which the median distance of all points along one axis is determined, grouping the points into those on either side of the median. This process is repeated for each dimension at each level of the tree before being recursively applied, until each level of the tree contains only two points. This enables the tree to be searched relatively quickly for nearest neighbors.

Although k-d trees are efficient for nearest-neighbor point searches, after computing reachability we will have sets of states. It is unclear how to extend k-d trees to do collision checking between sets that may have different sizes. 
The last idea we considered was using axis-aligned bounding-box (AABB) trees [4]. AABB trees provide a bounding-volume at each inner-node in the tree which that contains the volume of children of that node. They are often used for real-time $n$-to- $n$ collision detection in video games due to their flexibility and high-performance. Since they do support encoding sets of states, we can insert the reachable regions for each satellite into an $\mathrm{AABB}$ tree, and then efficiently check for collisions between sets.

Time-stepping strategy. The last optimization deals with the time-stepping strategy. Since we want to do analysis with a time bound on the order of days, ideally we would consider large time steps (intervals of time to be analyzed). However, large time steps will lead to large ranges of $\nu$ reachable for each satellite, which would leave to large boxes in Cartesian coordinates. This could cause false positives when checking for collisions. Using small time steps, however, would lead to a large number of steps that needs to be analyzed. For this reason, we propose to use a CEGAR-based variable time stepping strategy. We start with a minimum time step such as 0.1 second, and do a step of that size. If the step succeeds (no collisions are detected), we advance time and double the step size. If collisions are detected, we halve the step size and try again without advancing time. If the step size is ever reduced to below the minimum ( 0.1 seconds), we give up and output that a collision is likely possible.

\subsection{Evaluation}

We have developed custom code to perform the numerical simulations and leverage the problem structure described in the previous section. The goal was to perform preliminary analysis on the viability of the verification of this benchmark, and we have left a detailed comparison of existing algorithms and tools for nonlinear reachability analysis as a future research item.

In our Python-based implementation, we use scipy's integrate. RK45 method for satellite simulation, and the pyibex library to perform interval arithmetic. The measurements are performed on an Intel i5-5300U CPU running at $2.30 \mathrm{GHz}$ with $16 \mathrm{~GB}$ main memory. Since the satellite generation is random, the source code used for the benchmark should be included as an attachment with this paper.

We evaluate the proposed approach on two aspects. First, we measure the runtime of various aspects of the algorithm, comparing the previously-described brute force collision check versus the AABB tree method. Next, we analyze the effectiveness of the dynamic time-stepping strategy.

We analyze the system by generating uniformly random satellite parameters. We are concerned with satellites in low-earth orbit, so the satellite height in $\mathrm{km}, h$, is chosen from $[6951,7221]$. The satellite orbits we are interested are generally round so we use an eccentricity $e$ in the range [0,0.004]. The angles $\nu, \omega$, and $\Omega$ are all taken from the range $[0,2 \pi]$ and $i$ is taken from the range $[0, \pi]$.

First we compare the brute force search method versus the AABB tree approach. We using a small collision distance of 100 meters (in the infinity norm), with small initial $\nu$ uncertainty of 0.0001. With these parameters, no collisions should exist in the time frames analyzed.

In the 100 satellite case, with a reach time bound of one hour, the brute force method took 6.9 seconds, whereas the AABB tree approach used 4.2 seconds. Increasing the number of satellites to 1000, and decreasing the reach time bound to one minute, the brute force method needed 28.3 seconds (about half of real-time), whereas the AABB approach completed 
Table 3: Computation Time Comparison

\begin{tabular}{lllll}
\hline Number of Satellites & Time Bound Brute Force & AABB (dynamic) & AABB (static) \\
\hline 100 & $1 \mathrm{hr}$ & $6.9 \mathrm{~s}$ & $4.2 \mathrm{~s}$ & \\
1000 & $1 \mathrm{~min}$ & $28.3 \mathrm{~s}$ & $3.7 \mathrm{~s}$ & $80 \mathrm{~s}$ \\
5000 & $10 \mathrm{~s}$ & $260 \mathrm{~s}$ & $13.9 \mathrm{~s}$ & \\
\hline
\end{tabular}

in 3.7 seconds. Finally, with a reach time bound of 10 seconds and 5000 satellites, the brute force method required 260 seconds, whereas the AABB tree approach needed 13.9 seconds. As expected, the AABB method was significantly faster, but still it was still slower than real-time for the $n=5000$ case.

To analyze the effect of the dynamic time-stepping strategy, we used the AABB method with 1000 satellites for one minute of reach time. A static stepping strategy which always used the minimum step of 0.1 seconds required 80 seconds to complete, compared with 3.7 seconds with the dynamic time stepping approach. In this case, without dynamic times steps, the method runs much slower than real-time. Further, in the dynamic case, the majority of the runtime, $80 \%$, is spent in the interval arithmetic library constructing the boxes of reachable Cartesian coordinates. This means that AABB trees are sufficiently fast so the collision checking is not the bottleneck. The results are summarized in Table 3 .

Overall, our evaluation shows that reachability analysis, with the enhancements described, can be viable for the space debris collision avoidance problem with a few thousand objects. If we want to scale to tens of thousands or hundreds of thousands of objects, however, further speedups are needed. In the 5000 satellite case, all our current methods were slower than real-time, which is unusable in practice. One idea for speedup would be to consider different time-steps for different objects. Currently, if there is any collision, all objects get rolled back and a smaller time-step is attempted. As we add more objects, the potential for collisions will increase, and so time-step rollbacks will become more common. We will investigate per-object time-steps in future work.

The provided code can be used to create nonlinear benchmarks with any number of dimensions. The property is always $n$-to- $n$ collision avoidance, but a second parameter is the collision radius in terms of infinity-norm distance. For just evaluating the runtime, a small initial uncertainty in $\nu$, and a small collision radius should be used, so that no collisions occur up to the time bound. To generate instances with collisions, the collision radius and/or the initial uncertainty can be increased.

\section{Conclusion}

In this paper, we presented a space debris collision detection problem and initial analysis approach. In the current setup, we considered orbital dynamics with a single variable, the angular position $\nu$. An immediate extension, then, would be to consider ranges for the other orbital parameters, corresponding to errors in sensing space objects. For fixed parameter ranges, we might directly be able to use the existing interval arithmetic approach without modification, simply using the interval ranges when computing the Cartesian coordinates. A more realistic setup, however, would give these parameters their own dynamics that spread out over time. In particular, we could consider the semi-major axis and eccentricity to be variables with a small initial uncertainty that can slowly diverge with a known minimum and maximum rate. This would mean we no longer have 1-d systems where we can use simulations for reachability, but instead we would need to use more sophisticated nonlinear reachability methods [7]. 
Although we focused on orbital dynamics, many of the problems encountered are more general. Swarm robotics, for example, also have $n$-to- $n$ collision avoidance requirements. Although these system are not 1-dimensional, they do consist of isolated systems that generally fly independently, and so decomposition may still be possible.

\section{References}

[1] S. Alfano. Determining satellite close approaches, part 2. Journal of the Astronautical Sciences, 42:143-152, 1994.

[2] K. T. Alfriend, M. R. Akella, J. Frisbee, J. L. Foster, D.-J. Lee, and M. Wilkins. Probability of collision error analysis. Space Debris, 1(1):21-35, 1999.

[3] S. Bak, S. Bogomolov, T. A. Henzinger, T. T. Johnson, and P. Prakash. Scalable static hybridization methods for analysis of nonlinear systems. In International Conference on Hybrid Systems: Computation and Control, 2016.

[4] G. v. d. Bergen. Efficient collision detection of complex deformable models using aabb trees. Journal of Graphics Tools, 2(4):1-13, 1997.

[5] K. Chan. Spacecraft collision probability for long-term encounters. Advances in the Astronautical Sciences, 116(1):767-784, 2003.

[6] N. Chan and S. Mitra. Verifying safety of an autonomous spacecraft rendezvous mission. In G. Frehse and M. Althoff, editors, ARCH17. 4th International Workshop on Applied Verification of Continuous and Hybrid Systems, volume 48 of EPiC Series in Computing. EasyChair, 2017.

[7] X. Chen, E. Abraham, and S. Sankaranarayanan. Taylor model flowpipe construction for nonlinear hybrid systems. Real-Time Systems Symposium, 2012.

[8] X. Chen, M. Althoff, and F. Immler. Arch-comp17 category report: Continuous systems with nonlinear dynamics. In G. Frehse and M. Althoff, editors, ARCH17. 4th International Workshop on Applied Verification of Continuous and Hybrid Systems, volume 48 of EPiC Series in Computing, pages 160-169. EasyChair, 2017.

[9] T. Dang. Verification et synthese des systemes hybrides. PhD thesis, INPG, oct 2000.

[10] K. J. DeMars, Y. Cheng, and M. K. Jah. Collision probability with gaussian mixture orbit uncertainty. Journal of Guidance, Control, and Dynamics, 37(3):979-985, 2014.

[11] J. Green. Compositional bounded reachability using time partitioning and abstraction, 2012.

[12] T. A. Henzinger, P.-H. Ho, and H. Wong-Toi. Algorithmic analysis of nonlinear hybrid systems. IEEE transactions on automatic control, 43(4):540-554, 1998.

[13] S. A. Hildreth and A. Arnold. Threats to u.s. national security interests in space: Orbital debris mitigation and removal. Technical report, Congressional Research Service, 2014.

[14] B. HomChaudhuri, M. Oishi, M. Shubert, M. Baldwin, and R. S. Erwin. Computing reach-avoid sets for space vehicle docking under continuous thrust. In Decision and Control (CDC), 2016 IEEE 55th Conference on, pages 3312-3318. IEEE, 2016.

[15] L. Jaulin. Applied interval analysis: with examples in parameter and state estimation, robust control and robotics, volume 1. Springer Science \& Business Media, 2001.

[16] C. Jewison and R. S. Erwin. A spacecraft benchmark problem for hybrid control and estimation. In Decision and Control (CDC), 2016 IEEE 55th Conference on, pages 3300-3305. IEEE, 2016.

[17] T. T. Johnson, J. Green, S. Mitra, R. Dudley, and R. S. Erwin. Satellite rendezvous and conjunction avoidance: Case studies in verification of nonlinear hybrid systems. In International Symposium on Formal Methods, pages 252-266. Springer, 2012.

[18] R. P. Patera. Space vehicle conflict-avoidance analysis. Journal of guidance, control, and dynamics, 30(2):492-498, 2007.

[19] W. E. Wiesel. Spaceflight Dynamics. Aphelion Press, Beavercreek, Ohio, 45434, 3 edition, 2010. 